\title{
APPLICATIONS OF ISOTOPE GEOCHEMISTRY TO RESEARCH ON CHINESE GLACIERS
}

\author{
by \\ Wang Ping* \\ (Lanzhou Institute of Glaciology and Cryopedology, Academia Sinica, Lanzhou 730000, China)
}

\begin{abstract}
We report trace element, tritium, and hydrochemical analyses for glaciers in mountain regions of western China, based on about 200 samples collected between 1980 and 1983. Conclusions are drawn about the origin of water vapour contributing to precipitation (local or otherwise) and about the contributions to glacier run-off (meltwater or precipitation), based on the tritium measurements.
\end{abstract}

\section{INTRODUCTION}

The glaciers studied are located in mountain areas, at altitudes of over $3000 \mathrm{~m}$, in western China and are listed in Table I. Snow samples were also collected from the Kunlun mountains, Fenghuoshan (K'un-lun mountains, Feng-huo-shan) and the Tanggula mountains, Qinghai-Xizang Plateau (T'ang-ku-la mountains, Ch'ing-hai - Hsi-tsang Plateau).

\section{RESULTS AND DISCUSSION}

1. Hydrological characteristics and trace elements

Our results show that the $\mathrm{pH}$ of melted snow and ice samples is approximately neutral (Table II) (Vilenskiy and Miklishanskiy 1976, Wang Ping and Liu Zhi 1982, Wang Ping and others 1983); however, a snow sample from Galongla glacier had a $\mathrm{pH}$ of 4.25 , which is unusually low for the area studied. The degree of mineralization of the samples was also low, samples from "Hars", Gonggashan, and Duoxiongla glaciers generally containing less than $15 \mathrm{mg} \mathrm{l}^{-1}$ of dissolved mineral salts. This shows that the snow and ice of Chinese glaciers are generally fresh and extremely soft.

Table III summarizes the measured concentrations of trace elements and heavy metals. Concentrations of $\mathrm{Mg}, \mathrm{Co}$, $\mathrm{Cr}, \mathrm{Sr}, \mathrm{Ni}, \mathrm{Cu}, \mathrm{Zn}, \mathrm{V}, \mathrm{Pb}, \mathrm{Ti}, \mathrm{Sn}, \mathrm{Hg}, \mathrm{Ag}$ and $\mathrm{Se}$ were all found to be below the maximum permissible levels for harmful metals in groundwater, as published by the Department of Health in China in 1963. However, the levels of $\mathrm{As}$ and $\mathrm{Cd}$ were found sometimes to exceed the maximum safe concentrations; for example, the levels of As in samples taken from Dunte flat-topped glacier and Zelonglong glacier were above $50 \mathrm{ppb}$, and samples from Zelonglong, Galongla, Namula, and Duoxiongla glaciers showed Cd levels in excess of $10 \mathrm{ppb}$ (the National Standard).

\section{*Wade-Giles: Wang P'ing}

House Editor's note: in this paper, when a Chinese name is used, the Pinyin form of transliteration is given first, followed by the Wade-Giles form in round brackets (for the convenience of bibliographers who are accustomed to this form). However, Table I lists these forms for glaciers and their geographical locations (including two instances where anglicized forms of Chinese names of glaciers are commonly used, even in China), and the list at the end of the paper includes transliterations for all references in Chinese (authors, titles of articles, periodicals) together with English translations. In these cases, therefore, only the Pinyin form is given in the text.

\section{Impurity enrichment factors}

Table IV shows measured enrichment factors for major impurities in Chinese mountain glaciers (Boutron and Lorius 1977, Wang Ping and Luo Hongzhen 1980, Luo Hongzhen 1983, Wang Ping 1983). The order of enrichment of the major ions was found to be:

"Hars" glacier $\mathrm{Na}>\mathrm{K}>\mathrm{Mg}>\mathrm{Ca}$ or $\mathrm{Mg}>\mathrm{K}>\mathrm{Na}>\mathrm{Ca}$ Tianshan Mountains $\mathrm{Mg}>\mathrm{Ca}>\mathrm{Na}>\mathrm{K}$ or $\mathrm{Ca}>\mathrm{Mg}>\mathrm{Na}>\mathrm{K}$

Dunte flat-topped glacier Gonggashan glacier $\mathrm{Mg}>\mathrm{Na}>\mathrm{Ca}>\mathrm{K}$ or $\mathrm{Na}>\mathrm{Mg}>\mathrm{Ca}>\mathrm{K}$ Zelonglong glacier $\mathrm{Mg}>\mathrm{Ca}>\mathrm{Na}>\mathrm{K}$

Galongla glacier $\quad \mathrm{Na}>\mathrm{Mg}>\mathrm{K}>\mathrm{Ca}$

Namula and

Duoxiongla glaciers $\mathrm{Mg}>\mathrm{Ca}>\mathrm{K}>\mathrm{Na}$ or $\mathrm{K}>\mathrm{Na}>\mathrm{Ca}>\mathrm{Mg}$

Zhongrongbu glacier $\mathrm{Ca}>\mathrm{Na}>\mathrm{Mg}>\mathrm{K}$

Yulongshan glacier $\mathrm{Na}>\mathrm{Mg}>\mathrm{Ca}>\mathrm{K}$

Kunlun, Fenghuoshan

and Tanggula Mts $\mathrm{Mg}>\mathrm{Na}>\mathrm{K}>\mathrm{Ca}$ or $\mathrm{Na}>\mathrm{Ca}>\mathrm{Mg}>\mathrm{K}$

This shows that the pathways of water replenishment vary with locality in the mountainous regions of China, and that it is mainly controlled by the high atmospheric oceanic water vapour.

\section{Tritium measurements}

Tritium measurements can be used to estimate the annual accumulation and mean accumulation ratios (Boutron and Lorius 1977, Wang Ping and others 1984). We found environmental tritium levels in the range from 13 to 196 TU in samples of precipitation from the Altay, Tianshan, Qilian, Gonggashan, Tanggula and Kunlun mountains and from the Nanjiabawa Peak area (Table V). We also determined mean annual accumulation ratios of $618 \mathrm{~mm} \mathrm{a}^{-1}$ (west Qiongtailan glacier), $645 \mathrm{~mm} \mathrm{a}^{-1}$ ("Hars" glacier), and $424 \mathrm{~mm} \mathrm{a}^{-1}$ ("No.1 glacier").

Tritium data can also be used to calculate the percentage of local vapour in the air and precipitation of glacial regions (Wang Ping and others 1984). Based on these results, local contributions were calculated:

$\begin{array}{ll}\text { Mt Youyi (Altay Mountains) } & 35 \% \\ \text { "No.1 glacier" } & 12.0 \%(1981) \\ \text { west Qiongtailan glacier } & 17.6 \% \\ \text { Badaishi glacier } & 30.5 \% \\ \text { Lenglongling glacier } & 40.6 \% \\ \text { Gonggashan glacier } & 13.5 \% \\ \text { Tanggula Mountains } & 40.0 \% \\ \text { Yulongshan glacier } & 23.0 \% \\ \text { Nanjibawa glaciers } & 22.3 \%\end{array}$

$15.8 \%(1982)$

"No.1 glacier"

Lenglongling glacier

Gonggashan glacier

Yulongshan glacier

Nanjibawa glacier

Finally, we can use tritium measurements to separate the different contributions to the total glacier run-off. Analysis of the tritium content at the source of Qiongtailan River, Mt Tuomer, by Wang Lilun (Wang Li-lun), Su Zhen (Su Chen) and Zhang Wenjing (Chang Wen-ching) in July 1978 shows that glacier meltwater accounts for $34 \%$ and precipitation for $66 \%$ of the total glacier run-off. Table VI shows results obtained by the present author for "No.1 glacier" at the source of the Urumqi River, Tianshan mountains (Urumchi River, T'ien Shan) at different times during 1983. 


\section{ACKNOWLEDGEMENTS}

I am grateful to comrades Su Zhen (Su Chen), Wang Lilun (Wang Li-lun), Zhang Wenjing (Chang Wen-ching), Sheng Wenkun (Sheng Wen-k'un), Luo Hongzhen (Lo Hung-chen), Lin Zidong (Lin Tzu-tung), Chang Xiaooxiao (Ch'ang Hsiao-hsiao), Zhu Shousen (Chu Shou-sen), Li Shude (Li Shu'te) and Shi Qinseng (Shih Ch'in-seng) for taking part in this work.

\section{REFERENCES}

Boutron C, Lorius C 1977 Trace element content in East Antarctica snow samples. International Association of Hydrological Sciences Publication 118 (General Assembly of Grenoble 1975-Isotopes and Impurities in Snow and Ice): $164-171$

Luo Hongzhen (Lo Hung-chen) 1983 Tian shan Wu-lu-mu-qi he-yuan yi-hao bing-chuan de shui-hua-xue te-zheng ( $T$ 'ien shan Wu-lu-mu-ch'i he-yuan yi-hao ping ch'uan te shui-hua-hsueh t'e-cheng [Hydrochemical features of "No.1 glacier" in the source region of Urumqi river, Tianshan]. Bingchuan Dongtu (Ping-ch'uan Tung-t'u) [Journal of Glaciology and Cryopedology] 5(2): 55-64

Vilenskiy V D, Miklishanskiy A Z 1976 Khimicheskiy sostav snezhnogo pokrova Vostochnoy Antarktidy [Chemical composition of the snow cover of the eastern Antarctic region]. Geokhimiya 1976(11): 1683-1690

Wang Ping (Wang P'ing) 1983 A-er-tai shan You-yi feng Ha-la-si bing-chuan xue hen-liang yuan-su fen-xi (A-erh-t'ai shan Yu-yi feng Ha-la-ssu ping-ch'uan hsueh hen-liang yuan-su fen-hsi) [Analysis of trace elements in snow and ice on Ha-la-si glacier of Mt Youyi in the Altay Mountains]. Bingchuan Dongtu (Ping-ch'uan Tung-t'u) [Journal of Glaciology and Cryopedology] 5(1): 63-70

Wang Ping (Wang P'ing), Liu Zhi (Liu Chih) 1982 A-er-tai shan You-yi feng di-qu bing, xue ji qi shou bing-chuan rong-shui bu-ji jing-liu zhong de wei-liang yuan-su han-liang (A-erh-t'ai shan Yu-yi feng ti-ch'ü ping, hsueh chi ch'i shou ping-ch'uan jung-shui pu-chi ching-liu chung te wei-liäng yuan-su han-liang) [Trace element contents in ice, snow and glacier meltwater run-off on the "Hars" glacier of Mt Youyi in the Altay Mountains]. Huanjing Kexue (Huan-ching K'e-hsueh) [Journal of Environment Science] 3(3): 33-35

Wang Ping (Wang P'ing), Luo Hongzhon (Lo Hung-chen) 1980 Tuo-mu-er-feng Xi-qiong-tai-lan bing-xue-zhong-de hen-liang yuan-su han-liang (T'o mu-erh-feng Hsi-ch'iung-t'ai-lan ping-hsueh-chung-te hen-liang yuan-su han-liang) [Trace element contents in snow and ice samples of west Qiongtailan glacier, Mt Tuomer]. Bingchuan Dongtu (zeng-kan) (Ping-ch'uan Tung-t'u (tseng-k'an) [Journal of Glaciology and Cryopedology (Additional Issue)] 2: 77-79

Wang Ping (Wang P'ing), Jiang Lujian (Chiang Lu-chien), Liu Zhi (Liu Chih), Zhu Yongping (Chu Yung-p'ing), Ni Tongwen (Ni T'ung-wen) 1983 Lanzhou shi jiang-shui suan-du he zhong-jin-shu yuan-su de ce-ding (Lan-chou shih chiang-shui suan-tu he chong-chin-shu yuan-su te ts'e-ting) [Determination of acidity and heavy metal elements in precipitated water of Lanzhou city]. Huanjing Kexue (Huan-ching K'e-hsieh) [Journal of Environment Science] 4(5): 61-62

Wang Ping (Wang P'ing), Luo Hongzhen (Lo Hung-chen), Lin Ruifen (Lin Jui-fen), Wei Keqin (Wei K'e-ch' in), Wang Zhixiang (Wang Chih-hsiang) 1984 A-er-tai shan Ha-la-si bing-chuan he Tian shan Wu-lu-mu-qi he-yuan Yi-hao bing-chuan-qu bing-xue zhong chuan han-liang de fen-xi (A-erh-t'ai shan Ha-la-ssu ping-ch'uan he T'ien shan Wu-lu-mu-ch'i he-yuan yi-hao ping-ch'uan-ch'ü ping-hsueh chung ch'uan han-liang te fen-hsi) [Tritium content of ice and snow samples in "Hars" glacier and "No.1 glacier" at the source of Urumqi river, Tianshan]. Kexue Tongbao (K'e-hsueh T'ung-pao) [Monthly Journal of Science] 29(1): 83-87

TABLE I. LIST OF GLACIERS STUDIED AND THEIR GEOGRAPHICAL LOCATIONS, WITH PINYIN AND WADE-GILES TRANSLITERATIONS

\begin{tabular}{|c|c|c|c|}
\hline $\begin{array}{l}\text { Name of glacier } \\
\text { (Pinyin) }\end{array}$ & $\begin{array}{l}\text { Geographical location } \\
\text { (Pinyin) }\end{array}$ & $\begin{array}{l}\text { Name of glacier } \\
\text { (Wade-Giles) }\end{array}$ & $\begin{array}{l}\text { Geographical location } \\
\text { (Wade-Giles) }\end{array}$ \\
\hline
\end{tabular}

\section{Ha-la-si}

["Hars"]

Haxileigen

Qiongtailan

Keqiqiaer

Yi-hao bing-chuan ["No.1 glacier"]

Badaishi

Dunte

Lenglongling

Gonggashan

Yulongshan

Zelonglong

Galongla

Namula

Duoxiongla

Rongrongbu
Mt Youyi,

Altay mountains

western Tianshan

Mt Tuomuer, Tianshan

southern slope of Mt Tuomuer

source of Urumqi river, Tianshan

Haerlikeshan,

Hami

Kakitushan, south-west Qilian Shan

eastern Qilian Shan

Hengduan mountains

northern Yunnan

a-la-ssu

["Hars"]

Ha-hsi-lei-ken

Hsi-ch'iung-t'ai-lan

K'e-ch'i-ch'ia-erh

Yi-hao ping-ch'uan

["No.1 glacier"]

Pa-tai-shih

Tun-t'e

Lenglung Ling

Kung-ka-shan

Yu-lung-shan

Tse-lung-lung

vicinity of

Nanjiabawa Peak,

Ximalaya mountains

Ka-lung-la

Na-mu-la

To-hsiung-la
You-yi-feng

Altay mountains

western T'ien Shan

T'o mu-erh-feng,

T'ien Shan

southern slope of

T'omu-erh-feng

source of Urumchi river, T'ien Shan

Ha-erh-li-k'e-shan, $\mathrm{Ha}-\mathrm{mi}$

Kakitu Shan, south-west Ch'i-lien Shan

eastern Ch'i-lien Shan

Heng-tuan mountains

northern Yun-nan

vicinity of

Nan-chia-pa-wa Peak,

Hsi-ma-la-ya mountains 
TABLE II. THE DEGREE OF MINERALIZATION AND HYDROCHEMICAL TYPE IN SNOW AND ICE SAMPLES

\begin{tabular}{|c|c|c|c|c|c|c|}
\hline Glacier & $\begin{array}{l}\text { Time of } \\
\text { collection }\end{array}$ & Altitude & $\mathrm{pH}$ & Type & $\begin{array}{l}\text { Degree of mineralization } \\
\qquad\left(\mathrm{mg} \mathrm{l}^{-1}\right)\end{array}$ & Hydrochemical type \\
\hline $\begin{array}{l}\text { "Hars" glacier, } \\
\text { Mt Youyi, } \\
\text { Altay } \\
\text { Mountains }\end{array}$ & $\begin{array}{ll}\text { July } & 1980 \\
\text { July } & 1980 \\
\text { Aug } & 1980 \\
\text { Aug } & 1980 \\
\text { Aug } & 1980 \\
\text { Aug } & 1980\end{array}$ & $\begin{array}{l}3200 \\
2800 \\
3300 \\
2800 \\
3000 \\
3200\end{array}$ & $\begin{array}{l}6.62 \\
6.16 \\
6.07 \\
6.60 \\
6.52 \\
6.24\end{array}$ & $\begin{array}{l}\text { snow } \\
\text { snow } \\
\text { snow } \\
\text { ice } \\
\text { ice } \\
\text { ice }\end{array}$ & $\begin{array}{r}15.35 \\
25.09 \\
23.82 \\
12.59 \\
9.49 \\
9.50\end{array}$ & $\begin{array}{l}\mathrm{Mg}^{2+}, \mathrm{Na}^{+}, \mathrm{HCO}_{3}^{-}, \mathrm{Cl}^{-} \\
\mathrm{Na}^{+}, \mathrm{Mg}^{2+}, \mathrm{HCO}_{3}^{-}, \mathrm{Cl}^{-} \\
\mathrm{Na}^{+}, \mathrm{Mg}^{2+}, \mathrm{HCO}_{3}^{-}, \mathrm{Cl}^{-} \\
\mathrm{Na}^{+}, \mathrm{Mg}^{2+}, \mathrm{HCO}_{3}^{-} \\
\mathrm{Na}^{+}, \mathrm{Mg}^{2+}, \mathrm{HCO}_{3}^{-}, \mathrm{Cl}^{-} \\
\mathrm{Mg}^{2+}, \mathrm{Na}^{+}, \mathrm{SO}_{4}^{2-}, \mathrm{Cl}^{-}\end{array}$ \\
\hline $\begin{array}{l}\text { Haxileiggen } \\
\text { glacier }\end{array}$ & June 1981 & 3600 & 6.80 & snow & 14.13 & $\mathrm{Mg}^{2+}, \mathrm{Ca}^{2+}, \mathrm{SO}_{4}^{2-}, \mathrm{HCO}_{3}^{-}$ \\
\hline $\begin{array}{l}\text { Mt Tuomuer } \\
\text { (northern) }\end{array}$ & $\begin{array}{l}\text { July } 1981 \\
\text { July } 1981\end{array}$ & $\begin{array}{l}4000 \\
4000\end{array}$ & $\begin{array}{l}6.70 \\
7.90\end{array}$ & $\begin{array}{l}\text { hail } \\
\text { snow }\end{array}$ & $\begin{array}{l}35.98 \\
25.32\end{array}$ & $\begin{array}{l}\mathrm{Ca}^{2+}, \mathrm{Mg}^{2+}, \mathrm{SO}_{4}^{2-} \\
\mathrm{Ca}^{2+}, \mathrm{Mg}^{2+}, \mathrm{SO}_{4}^{2-}, \mathrm{HCO}_{3}^{-}\end{array}$ \\
\hline $\begin{array}{l}\text { Mt Tuomuer } \\
\text { (southern) }\end{array}$ & $\begin{array}{l}\text { July } 1981 \\
\text { June } 1979 \\
\text { June } 1979 \\
\text { June } 1979\end{array}$ & $\begin{array}{l}3600 \\
5300 \\
5300 \\
5300\end{array}$ & $\begin{array}{r}6.9 \\
- \\
- \\
-\end{array}$ & $\begin{array}{l}\text { snow } \\
\text { snow } \\
\text { snow } \\
\text { snow }\end{array}$ & $\begin{array}{l}20.95 \\
39.15 \\
28.29 \\
14.46\end{array}$ & $\begin{array}{l}\mathrm{Ca}^{2+}, \mathrm{Mg}^{2+}, \mathrm{SO}_{4}^{2-} \\
\mathrm{Ca}^{2+}, \mathrm{Mg}^{2+}, \mathrm{HCO}_{3}^{-} \\
\mathrm{Ca}^{2+}, \mathrm{Mg}^{2+}, \mathrm{HCO}_{3}^{-} \\
\mathrm{Mg}^{2+}, \mathrm{Ca}^{2+}, \mathrm{HCO}_{3}^{-}\end{array}$ \\
\hline $\begin{array}{l}\text { No.1 glacier, } \\
\text { at the source } \\
\text { of Urumqi } \\
\text { River }\end{array}$ & $\begin{array}{l}\text { June } 1982 \\
\text { June } 1982 \\
\text { May } 1982\end{array}$ & $\begin{array}{l}3700 \\
4100\end{array}$ & $\begin{array}{l}6.34 \\
6.46\end{array}$ & $\begin{array}{l}\text { hail } \\
\text { rain } \\
\text { snow }\end{array}$ & $\begin{array}{r}41.14 \\
22.92 \\
9.25\end{array}$ & $\begin{array}{l}\mathrm{Ca}^{2+}, \mathrm{Mg}^{2+}, \mathrm{HCO}_{3}^{-} \\
\mathrm{Mg}^{2+}, \mathrm{Ca}^{2+}, \mathrm{HCO}_{3}^{-}, \mathrm{SO}_{4}^{2-} \\
\mathrm{Mg}^{2+}, \mathrm{Ca}^{2+}, \mathrm{HCO}_{3}^{-}, \mathrm{SO}_{4}^{2-}\end{array}$ \\
\hline $\begin{array}{l}\text { Badaishi } \\
\text { glacier }\end{array}$ & Aug 1981 & 3300 & 6.00 & snow & 25.59 & $\mathrm{Mg}^{2+}, \mathrm{Ca}^{2+}, \mathrm{SO}_{4}^{2-}, \mathrm{HCO}_{3}^{-}$ \\
\hline $\begin{array}{l}\text { Gonggashan } \\
\text { glacier }\end{array}$ & $\begin{array}{ll}\text { Aug } 1981 \\
\text { Aug } 1981\end{array}$ & $\begin{array}{l}6000 \\
6000\end{array}$ & $\begin{array}{l}6.12 \\
6.30\end{array}$ & $\begin{array}{l}\text { snow } \\
\text { ice }\end{array}$ & $\begin{array}{l}5.49 \\
9.40\end{array}$ & $\begin{array}{l}\mathrm{Mg}^{2+}, \mathrm{Na}^{+}, \mathrm{Cl}^{-}, \mathrm{HCO}_{3}^{-} \\
\mathrm{Mg}^{2+}, \mathrm{Na}^{+}, \mathrm{HCO}_{3}^{-}, \mathrm{Cl}^{-}\end{array}$ \\
\hline $\begin{array}{l}\text { Dunte } \\
\text { flattopped } \\
\text { glacier, } \\
\text { Kakitushan }\end{array}$ & $\begin{array}{ll}\text { Aug } & 1981 \\
\text { Aug } & 1981 \\
\text { Aug } & 1981 \\
\text { Aug } & 1981\end{array}$ & $\begin{array}{l}5000 \\
5200 \\
5320 \\
5300\end{array}$ & $\begin{array}{l}7.70 \\
6.65 \\
6.70 \\
7.00\end{array}$ & $\begin{array}{l}\text { ice } \\
\text { snow } \\
\text { snow } \\
\text { snow }\end{array}$ & $\begin{array}{r}166.98 \\
33.82 \\
31.12 \\
33.20\end{array}$ & $\begin{array}{l}\mathrm{Ca}^{2+}, \mathrm{Mg}^{2+}, \mathrm{HCO}_{3}^{-} \\
\mathrm{Na}^{+}, \mathrm{Mg}^{2+}, \mathrm{HCO}_{3}^{-} \\
\mathrm{Na}^{+}, \mathrm{Mg}^{2+}, \mathrm{HCO}_{3}^{-} \\
\mathrm{Mg}^{2+}, \mathrm{Na}^{+}, \mathrm{Cl}^{-}, \mathrm{HCO}_{3}^{-}\end{array}$ \\
\hline $\begin{array}{l}\text { Lenglongling } \\
\text { glacier }\end{array}$ & $\begin{array}{l}\text { June } 1963 \\
\text { July } 1982\end{array}$ & $\begin{array}{l}4100 \\
4300\end{array}$ & $\overline{6.43}$ & $\begin{array}{l}\text { snow } \\
\text { snow }\end{array}$ & $\begin{array}{l}32.47 \\
16.62\end{array}$ & $\begin{array}{l}\mathrm{Ca}^{2+}, \mathrm{Mg}^{2+}, \mathrm{HCO}_{3}^{-} \\
\mathrm{Ca}^{2+}, \mathrm{Mg}^{2+}, \mathrm{HCO}_{3}^{-}\end{array}$ \\
\hline $\begin{array}{l}\text { Zelonglong } \\
\text { glacier }\end{array}$ & $\begin{array}{ll}\text { Sept } & 1982 \\
\text { Sept } 1982\end{array}$ & $\begin{array}{l}3550 \\
3700\end{array}$ & $\begin{array}{l}6.68 \\
5.58\end{array}$ & $\begin{array}{r}\text { rain } \\
\text { ice }\end{array}$ & $\begin{array}{l}28.78 \\
21.70\end{array}$ & $\begin{array}{l}\mathrm{Mg}^{2+}, \mathrm{Ca}^{2+}, \mathrm{HCO}_{3}^{-} \\
\mathrm{Mg}^{2+}, \mathrm{Na}^{+}, \mathrm{HCO}_{3}^{-}\end{array}$ \\
\hline $\begin{array}{l}\text { Galongla } \\
\text { glacier }\end{array}$ & $\begin{array}{l}\text { Aug } 1982 \\
\text { Aug } 1982\end{array}$ & $\begin{array}{l}3640 \\
3700\end{array}$ & $\begin{array}{l}4.25 \\
6.26\end{array}$ & $\begin{array}{l}\text { snow } \\
\text { ice }\end{array}$ & $\begin{array}{l}21.71 \\
39.36\end{array}$ & $\begin{array}{l}\mathrm{Na}^{+}, \mathrm{Mg}^{2+}, \mathrm{Cl}^{-}, \mathrm{SO}_{4}^{2-} \\
\mathrm{Ca}^{2+}, \mathrm{SO}_{4}^{2-}, \mathrm{HCO}_{3}^{-}\end{array}$ \\
\hline $\begin{array}{l}\text { Duoxiongla } \\
\text { glacier }\end{array}$ & $\begin{array}{l}\text { Sept } 1982 \\
\text { Sept } 1982\end{array}$ & $\begin{array}{l}4100 \\
4100\end{array}$ & $\begin{array}{l}5.50 \\
5.47\end{array}$ & $\begin{array}{l}\text { firn } \\
\text { firn }\end{array}$ & $\begin{array}{l}7.64 \\
3.08\end{array}$ & $\begin{array}{l}\mathrm{Mg}^{2+}, \mathrm{HCO}_{3}^{-}, \mathrm{Cl}^{-} \\
\mathrm{Mg}^{2+}, \mathrm{Ca}^{2+}, \mathrm{HCO}_{3}^{-}, \mathrm{Cl}^{-}\end{array}$ \\
\hline
\end{tabular}




\begin{tabular}{|c|c|c|c|c|c|c|c|c|c|c|c|c|c|c|c|c|c|c|c|}
\hline \multirow{2}{*}{ Glacier } & \multirow{2}{*}{$\begin{array}{l}\text { Sampling } \\
\text { date }\end{array}$} & \multirow{2}{*}{$\begin{array}{l}\text { Altitude } \\
\text { (m) }\end{array}$} & \multirow{2}{*}{ Type } & \multicolumn{16}{|c|}{$\mathrm{ppb}$} \\
\hline & & & & $\mathrm{Mg}$ & Co & $\mathrm{Cr}$ & $\mathrm{Sr}$ & $\mathrm{Ni}$ & $\mathrm{Cu}$ & $\mathrm{Zn}$ & V & $\mathrm{Pb}$ & As & $\mathrm{Cd}$ & $\mathrm{Ti}$ & Sn & $\mathrm{Hg}$ & $\mathrm{Ag}$ & $\mathrm{Se}$ \\
\hline \multirow{6}{*}{$\begin{array}{l}\text { "Hars" glacier, } \\
\text { Mt Youyi, } \\
\text { Altay } \\
\text { Mountains }\end{array}$} & July 1980 & 3200 & snow & & 171.7 & 10.7 & 2.1 & 2.4 & 13.9 & 10.1 & 11.8 & - & - & 0.3 & & & & & \\
\hline & July 1980 & 3000 & ice & & 159.0 & 8.3 & 6.5 & 5.3 & 11.0 & 9.1 & - & - & - & 0.3 & & & & & \\
\hline & July 1980 & 2800 & rain & & 45.0 & 1.0 & 4.0 & 0.6 & 0.9 & - & - & - & - & 0.3 & & & & & \\
\hline & July 1980 & 1900 & rain & & 45.0 & 1.0 & 1.7 & 0.6 & 0.9 & - & - & - & - & 0.3 & & & & & \\
\hline & July 1980 & 480 & river & & 38.6 & - & 33.7 & - & 6.8 & - & - & - & 16.0 & 0.3 & & & & & \\
\hline & July 1980 & 1300 & & & - & - & 47.3 & - & - & - & - & - & - & 0.3 & & & & & \\
\hline \multirow{6}{*}{$\begin{array}{l}\text { Glaciers of } \\
\text { Tianshan } \\
\text { Mountains }\end{array}$} & July 1980 & 4000 & snow & & 0.13 & 1.5 & & & 0.9 & 11.0 & & & & 0.9 & & 0.38 & & & \\
\hline & July 1980 & 4000 & snow & & - & - & 14.2 & - & 0.5 & 3.6 & - & - & 11.8 & 0.3 & - & 1.55 & & & \\
\hline & Aug 1980 & 4000 & ice & & 0.13 & 1.4 & & & 0.8 & 27.0 & & & & 0.9 & & 0.50 & & & \\
\hline & Aug 1982 & 3700 & hail & 29.4 & 83.2 & 17.2 & 41.1 & - & 29.8 & 24.2 & 12.0 & 9.7 & - & 9.3 & 0.1 & 6.1 & 11.7 & 17.4 & 16.8 \\
\hline & June 1982 & 3700 & rain & 26.0 & 64.2 & 11.2 & 21.6 & - & 20.7 & 72.9 & 10.9 & 6.4 & - & 7.7 & - & 3.0 & 11.0 & 15.0 & 11.9 \\
\hline & May 1982 & 4200 & snow & 8.3 & 76.8 & 12.0 & 11.8 & - & 22.5 & 80.1 & 12.1 & 6.7 & - & 8.0 & 0.4 & 4.4 & 10.7 & 16.3 & 11.4 \\
\hline \multirow{4}{*}{$\begin{array}{l}\text { Dunte } \\
\text { flattopped } \\
\text { glacier, } \\
\text { Kakitushan }\end{array}$} & Aug 1981 & 5000 & ice & & - & - & 82.0 & - & - & - & 0.3 & - & - & - & - & - & & - & \\
\hline & Aug 1981 & 5100 & snow & & - & 1.6 & 6.5 & 4.0 & 11.5 & 3.8 & - & - & 46.7 & 0.9 & 6.5 & - & & - & \\
\hline & Aug 1981 & 5300 & snow & & - & - & 9.4 & - & 3.6 & 3.2 & - & - & - & 1.2 & - & - & & - & \\
\hline & Aug 1981 & 5200 & snow & & 0.37 & - & 3.3 & 77.2 & 28.0 & 9.5 & 9.4 & - & 92.1 & 12.8 & 1.1 & 4.7 & & 23.1 & \\
\hline $\begin{array}{l}\text { Lenglongling } \\
\text { glacier }\end{array}$ & Aug 1982 & 4300 & snow & 21.0 & 90.3 & 13.0 & 8.2 & - & 23.8 & 43.2 & 12.6 & 4.2 & - & 9.3 & 0.5 & 3.0 & 12.6 & 17.6 & 13.6 \\
\hline \multirow{2}{*}{$\begin{array}{l}\text { Gonggashan } \\
\text { glacier }\end{array}$} & Aug 1981 & 6000 & snow & & 0.04 & 28.5 & 0.8 & - & 20.5 & - & 5.5 & - & - & 0.3 & - & - & & 22.6 & \\
\hline & Aug 1981 & 6000 & ice & & 0.01 & 6.7 & - & - & 9.2 & - & - & - & - & 0.3 & - & - & & 19.6 & \\
\hline \multirow{5}{*}{$\begin{array}{l}\text { Zelonglong } \\
\text { glacier }\end{array}$} & Sept 1982 & 3700 & ice & 27.0 & 97.3 & 9.7 & 2.2 & 9.3 & 23.3 & 64.4 & 11.8 & 17.6 & - & 11.0 & 2.5 & 5.8 & 11.8 & 20.1 & 18.3 \\
\hline & Sept 1982 & 3500 & ice & 16.0 & 26.3 & 6.2 & 0.8 & - & 17.6 & 60.2 & 6.7 & 9.9 & - & 7.5 & - & 4.3 & 6.6 & 8.7 & 15.9 \\
\hline & Sept 1982 & 3550 & rain & 21.0 & 91.1 & 12.6 & 13.4 & 11.6 & 62.7 & 33.8 & 11.3 & 24.5 & 32.8 & 13.0 & 0.7 & 9.9 & 11.7 & 18.8 & 25.4 \\
\hline & Sept 1982 & 2820 & river & 41.9 & 107.6 & 11.6 & 72.3 & 9.9 & 21.7 & 73.3 & 12.0 & 18.1 & 135.0 & 11.9 & 0.8 & 13.8 & 12.8 & 19.9 & 32.1 \\
\hline & Oct 1982 & 2850 & spring & 59.8 & 166.6 & 16.7 & 147.6 & 25.1 & 24.0 & 87.1 & 15.8 & 32.7 & 508.2 & 19.2 & 1.5 & 30.0 & 16.7 & 24.6 & 63.0 \\
\hline \multirow{2}{*}{$\begin{array}{l}\text { Galongla } \\
\text { glacier }\end{array}$} & Aug 1982 & 3700 & ice & 19.4 & 91.7 & 11.5 & 3.9 & 1.5 & 30.8 & 71.5 & 12.1 & 14.2 & - & 11.4 & 0.5 & 6.5 & 12.7 & 19.4 & 17.9 \\
\hline & Aug 1982 & 3640 & snow & 31.3 & 108.4 & 14.6 & 2.3 & - & 39.1 & 268.9 & 15.1 & 13.2 & - & 11.9 & 1.1 & 6.1 & 14.6 & 22.2 & 19.9 \\
\hline \multirow{3}{*}{$\begin{array}{l}\text { Namula } \\
\text { glacier }\end{array}$} & Oct 1982 & 4600 & ice & 12.0 & 56.3 & 9.3 & 1.1 & - & 20.6 & 208.0 & 9.8 & 8.5 & - & 8.3 & - & 3.6 & 9.6 & 13.7 & 11.8 \\
\hline & Oct 1982 & 4600 & snow & 24.1 & 85.5 & 11.8 & 2.3 & - & 23.8 & 238.6 & 11.9 & 11.7 & - & 11.0 & 0.5 & 5.0 & 12.2 & 19.3 & 17.8 \\
\hline & Oct 1982 & 4130 & lake & 33.4 & 88.6 & 13.3 & 14.8 & - & 22.5 & 59.5 & 12.7 & 10.5 & - & 10.1 & 2.4 & 8.6 & 12.4 & 17.7 & 22.5 \\
\hline \multirow{2}{*}{$\begin{array}{l}\text { Duoxiongla } \\
\text { glacier }\end{array}$} & Sept 1982 & 4100 & snow & 28.4 & 81.6 & 8.8 & 0.2 & 4.2 & 21.4 & 63.9 & 9.5 & 15.9 & - & 9.7 & 0.4 & 4.9 & 9.9 & 16.0 & 16.6 \\
\hline & Sept 1982 & 4100 & snow & 35.5 & 88.0 & 11.2 & 0.2 & - & 21.6 & 65.2 & 12.3 & 14.8 & - & 12.1 & 0.2 & 6.7 & 12.8 & 19.3 & 18.6 \\
\hline \multirow{3}{*}{$\begin{array}{c}\text { Qinghai- } \\
\text { Xizang } \\
\text { Plateau }\end{array}$} & July 1980 & 4800 & snow & & 0.2 & 10.5 & 57.7 & 0.6 & 6.2 & - & 10.5 & & - & 0.3 & - & & & & \\
\hline & Aug 1982 & 4800 & snow & 22.0 & 98.2 & 13.6 & 2.4 & - & 22.9 & 60.3 & 13.7 & 12.0 & - & 10.1 & 0.9 & 6.8 & 13.1 & 20.6 & 17.7 \\
\hline & Sept 1982 & 4700 & snow & 19.2 & 92.6 & 12.6 & 15.7 & - & 25.9 & 106.6 & 12.8 & 11.6 & - & 10.8 & 0.6 & 6.1 & 12.6 & 19.9 & 15.8 \\
\hline \multirow{2}{*}{$\begin{array}{l}\text { Yulongshan } \\
\text { glacier }\end{array}$} & July 1982 & 4500 & firn & 12.2 & 37.2 & - & - & 10.4 & 27.0 & - & 2.5 & 26.9 & - & 15.5 & 4.6 & 9.7 & 24.4 & - & 18.3 \\
\hline & Aug 1982 & 4500 & snow & 11.6 & 30.2 & - & - & 3.4 & 20.8 & - & 1.9 & 16.7 & - & 9.8 & 3.5 & 4.1 & 18.7 & - & 5.9 \\
\hline
\end{tabular}


TABLE IV. ELEMENT ENRICHMENT FACTORS IN SNOW AND ICE

\begin{tabular}{|c|c|c|c|c|c|c|c|c|}
\hline \multirow[b]{2}{*}{ Glacier } & \multirow{2}{*}{$\begin{array}{l}\text { Sampling } \\
\text { time }\end{array}$} & \multirow{2}{*}{$\begin{array}{l}\text { Altitude } \\
\text { (m) }\end{array}$} & \multirow{2}{*}{$\begin{array}{l}\text { Type of } \\
\text { samples }\end{array}$} & \multicolumn{4}{|c|}{ Enrichment factor (EF/A1) } & \multirow{2}{*}{ Enrichment order } \\
\hline & & & & $\mathrm{Na}$ & $\mathrm{Mg}$ & $\mathrm{K}$ & $\mathrm{Ca}$ & \\
\hline \multirow{6}{*}{$\begin{array}{l}\text { "Hars" glacier, } \\
\text { Mt Youyi, } \\
\text { Altay } \\
\text { Mountains }\end{array}$} & Aug 1980 & 3380 & snow & 110.2 & 55.7 & 86.9 & 51.5 & $\mathrm{Na}>\mathrm{K}>\mathrm{Mg}>\mathrm{Ca}$ \\
\hline & July 1980 & 3200 & snow & 15.8 & 55.7 & 17.3 & 11.2 & $\mathrm{Mg}>\mathrm{K}>\mathrm{Na}>\mathrm{Ca}$ \\
\hline & July 1980 & 3200 & snow & 10.1 & 41.5 & 26.8 & 9.8 & $\mathrm{Mg}>\mathrm{K}>\mathrm{Na}>\mathrm{Ca}$ \\
\hline & July 1980 & 2800 & snow & 603.6 & 416.2 & 515.6 & 149.5 & $\mathrm{Na}>\mathrm{K}>\mathrm{Mg}>\mathrm{Ca}$ \\
\hline & July 1980 & 3200 & ice & 25.0 & 50.5 & 10.0 & 11.9 & $\mathrm{Mg}>\mathrm{Na}>\mathrm{Ca}>\mathrm{K}$ \\
\hline & Aug 1980 & 2600 & ice & 1.0 & 28.8 & 10.0 & 23.8 & $\mathrm{Mg}>\mathrm{Ca}>\mathrm{K}>\mathrm{Na}$ \\
\hline $\begin{array}{l}\text { Haxileigen } \\
\text { glacier }\end{array}$ & June 1981 & 3600 & snow & 5.3 & 37.4 & 3.5 & 25.4 & $\mathrm{Mg}>\mathrm{Ca}>\mathrm{Na}>\mathrm{K}$ \\
\hline \multirow{4}{*}{$\begin{array}{l}\text { Quiongtailan } \\
\text { glacier, } \\
\text { Mt Tuomuer }\end{array}$} & June 1978 & 4000 & snow & 1.6 & 2.3 & 0.3 & 5.1 & $\mathrm{Ca}>\mathrm{Mg}>\mathrm{Na}>\mathrm{K}$ \\
\hline & July 1981 & 3600 & snow & 2.6 & 16.6 & 0.7 & 33.9 & $\mathrm{Ca}>\mathrm{Mg}>\mathrm{Na}>\mathrm{K}$ \\
\hline & July 1981 & 4000 & snow & 4.5 & 23.6 & 4.2 & 30.5 & $\mathrm{Ca}>\mathrm{Mg}>\mathrm{Na}>\mathrm{K}$ \\
\hline & June 1978 & 4000 & ice & 49.8 & 54.1 & 13.0 & 101.3 & $\mathrm{Ca}>\mathrm{Mg}>\mathrm{Na}>\mathrm{K}$ \\
\hline \multirow{2}{*}{$\begin{array}{l}\text { No.1 glacier } \\
\text { at the source } \\
\text { of Urumqi }\end{array}$} & May 1982 & 4000 & snow & 3.9 & 16.7 & 2.8 & 13.4 & $\mathrm{Mg}>\mathrm{Ca}>\mathrm{Na}>\mathrm{K}$ \\
\hline & May 1982 & 4000 & snow & 8.8 & 25.6 & 2.9 & 17.6 & $\mathrm{Mg}>\mathrm{Ca}>\mathrm{Na}>\mathrm{K}$ \\
\hline $\begin{array}{c}\text { Badaishi } \\
\text { glacier }\end{array}$ & Aug 1981 & 3300 & snow & 23.3 & 49.4 & 22.2 & 30.2 & $\mathrm{Mg}>\mathrm{Ca}>\mathrm{Na}>\mathrm{K}$ \\
\hline \multirow{6}{*}{$\begin{array}{l}\text { Dunte } \\
\text { flattopped } \\
\text { glacier } \\
\text { Kakitushan }\end{array}$} & Aug 1981 & 5100 & snow & 15.3 & 20.2 & 11.6 & 6.3 & $\mathrm{Mg}>\mathrm{Na}>\mathrm{K}>\mathrm{Ca}$ \\
\hline & Aug 1981 & 5200 & snow & 109.2 & 34.4 & 30.5 & 32.4 & $\mathrm{Na}>\mathrm{Mg}>\mathrm{Ca}>\mathrm{K}$ \\
\hline & Aug 1981 & 5320 & snow & 48.2 & 43.4 & 5.7 & 13.1 & $\mathrm{Na}>\mathrm{Mg}>\mathrm{Ca}>\mathrm{K}$ \\
\hline & Aug 1981 & 5200 & snow & 158.3 & 74.2 & 133.5 & 55.8 & $\mathrm{Na}>\mathrm{K}>\mathrm{Mg}>\mathrm{Ca}$ \\
\hline & Aug 1981 & 5100 & snow & 5.0 & 21.6 & 1.1 & 11.1 & $\mathrm{Mg}>\mathrm{Ca}>\mathrm{Na}>\mathrm{K}$ \\
\hline & Aug 1981 & 5000 & ice & 4.8 & 17.0 & 3.9 & 28.5 & $\mathrm{Ca}>\mathrm{Mg}>\mathrm{Na}>\mathrm{K}$ \\
\hline $\begin{array}{l}\text { Lenglongling } \\
\text { glacier }\end{array}$ & Aug 1982 & 4300 & snow & 5.2 & 11.0 & 5.5 & 18.3 & $\mathrm{Ca}>\mathrm{Mg}>\mathrm{K}>\mathrm{Na}$ \\
\hline \multirow{2}{*}{$\begin{array}{l}\text { Gonggashan } \\
\text { glacier }\end{array}$} & Aug 1981 & 6000 & ice & 55.4 & 52.1 & 17.6 & 8.1 & $\mathrm{Na}>\mathrm{Mg}>\mathrm{K}>\mathrm{Ca}$ \\
\hline & Aug 1981 & 6000 & snow & 10.2 & 41.1 & 3.6 & 6.4 & $\mathrm{Mg}>\mathrm{Na}>\mathrm{Ca}>\mathrm{K}$ \\
\hline \multirow{3}{*}{$\begin{array}{l}\text { Zelonglong } \\
\text { glacier }\end{array}$} & Sept 1982 & 3550 & rain & 10.6 & 28.8 & 8.9 & 11.3 & $\mathrm{Mg}>\mathrm{Ca}>\mathrm{Na}>\mathrm{K}$ \\
\hline & Sept 1982 & 3700 & ice & 33.6 & 95.6 & 32.4 & 3.2 & $\mathrm{Mg}>\mathrm{Na}>\mathrm{K}>\mathrm{Ca}$ \\
\hline & Sept 1982 & 3500 & ice & 43.9 & 58.1 & 32.9 & 34.8 & $\mathrm{Mg}>\mathrm{Na}>\mathrm{Ca}>\mathrm{K}$ \\
\hline \multirow{2}{*}{$\begin{array}{l}\text { Galongla } \\
\text { glacier }\end{array}$} & Aug 1982 & 3640 & snow & 57.4 & 47.9 & 26.1 & 4.7 & $\mathrm{Na}>\mathrm{Mg}>\mathrm{K}>\mathrm{Ca}$ \\
\hline & Aug 1982 & 2700 & ice & 3.6 & 9.4 & 28.7 & 26.6 & $\mathrm{~K}>\mathrm{Ca}>\mathrm{Mg}>\mathrm{Na}$ \\
\hline \multirow{2}{*}{$\begin{array}{r}\text { Namula } \\
\text { glacier }\end{array}$} & Oct 1982 & 4600 & snow & 73.0 & 23.6 & 23.1 & 6.2 & $\mathrm{Mg}>\mathrm{K}>\mathrm{Ca}>\mathrm{Na}$ \\
\hline & Oct 1982 & 4600 & ice & 695.2 & 34.0 & 297.2 & 1.1 & $\mathrm{Mg}>\mathrm{Ca}>\mathrm{K}>\mathrm{Na}$ \\
\hline \multirow{2}{*}{$\begin{array}{l}\text { Duoxiongla } \\
\text { glacier }\end{array}$} & Sept 1982 & 4100 & firn & 9.7 & 42.4 & 18.6 & 24.3 & $\mathrm{Mg}>\mathrm{Ca}>\mathrm{K}>\mathrm{Na}$ \\
\hline & Sept 1982 & 4100 & firn & 9.0 & 7.4 & 18.3 & 7.5 & $\mathrm{~K}>\mathrm{Na}>\mathrm{Ca}>\mathrm{Mg}$ \\
\hline $\begin{array}{l}\text { Rongbuk } \\
\text { glacier }\end{array}$ & 1964 & & snow & 19.3 & 5.0 & 4.8 & 28.3 & $\mathrm{Ca}>\mathrm{Na}>\mathrm{Mg}>\mathrm{K}$ \\
\hline
\end{tabular}


TABLE V. TRITIUM CONTENT IN SNOWFALL FOR MOUNTAIN AREAS

\begin{tabular}{|c|c|c|c|c|c|c|c|}
\hline \multirow[b]{2}{*}{ Glacier } & \multirow{2}{*}{$\begin{array}{l}\text { Time of } \\
\text { collection }\end{array}$} & \multirow{2}{*}{$\begin{array}{l}\text { Altitude } \\
\text { (m) }\end{array}$} & \multirow{2}{*}{$\begin{array}{l}\text { Type of } \\
\text { samples }\end{array}$} & \multicolumn{2}{|c|}{ Position } & \multicolumn{2}{|c|}{ Tritium content (TU) } \\
\hline & & & & Latitude & Longitude & Content & Mean \\
\hline \begin{tabular}{|l} 
"Hars" \\
glacier
\end{tabular} & Aug 1980 & 3380 & snowfall & $49^{\circ} 07$ N & $87^{\circ} 47 \mathrm{I}$ & & $102.8^{*}$ \\
\hline $\begin{array}{l}\text { No.1 } \\
\text { glacier } \\
\text { at the } \\
\text { source } \\
\text { of Urumqi } \\
\text { River }\end{array}$ & \begin{tabular}{|ll} 
May- \\
Sept & 1980 \\
Aug & 1983 \\
Aug & 1983 \\
& \\
Aug & 1983 \\
Aug & 1983 \\
Aug & 1983 \\
Aug & 1983
\end{tabular} & $\begin{array}{l}4100 \\
4100 \\
4100 \\
\\
4100 \\
4100 \\
4100 \\
4100\end{array}$ & snowfall & $43^{\circ} 06^{\prime} \mathrm{N}$ & $86^{\circ} 49^{\prime} \mathrm{E}$ & $\begin{array}{r}113 \\
82 \\
\\
76 \\
118 \\
120 \\
95\end{array}$ & $106.7^{*}$ \\
\hline \multirow[t]{2}{*}{$\begin{array}{l}\text { Gonggashan } \\
\text { glacier }\end{array}$} & $\begin{array}{ll}\text { Sept } & 1982 \\
\text { Sept } & 1982 \\
\text { Sept } & 1982 \\
\text { Sept } & 1982\end{array}$ & $\begin{array}{l}5180 \\
5180 \\
5180 \\
5180\end{array}$ & firn & \multirow[t]{2}{*}{$29^{\circ} 36^{\prime} \mathrm{N}$} & \multirow[t]{2}{*}{$101^{\circ} 56^{\prime} \mathrm{E}$} & $\begin{array}{l}60 \\
19 \\
47 \\
26\end{array}$ & \multirow[t]{2}{*}{33.0} \\
\hline & Sept 1982 & 4100 & snowfall & & & 13 & \\
\hline $\begin{array}{l}\text { Region of } \\
\text { Nanjibawa } \\
\text { Peak }\end{array}$ & $\begin{array}{ll}\text { Oct } & 1982 \\
\text { Oct } & 1982 \\
\text { Oct } & 1982 \\
& \\
\text { Oct } & 1982 \\
\text { Oct } & 1982 \\
\text { Oct } & 1982\end{array}$ & $\begin{array}{l}4700 \\
4700 \\
4700 \\
\\
4700 \\
4500 \\
4500\end{array}$ & snow & $29^{\circ} 38^{\prime} \mathrm{N}$ & $95^{\circ} 10^{\prime} \mathrm{E}$ & $\begin{array}{l}34 \\
27 \\
23 \\
\\
\\
31 \\
15 \\
23\end{array}$ & 25.5 \\
\hline \multirow{2}{*}{$\begin{array}{l}\text { Yulongshan } \\
\text { glacier }\end{array}$} & \begin{tabular}{|ll} 
July & 1983 \\
July & 1983 \\
Aug & 1982 \\
\end{tabular} & $\begin{array}{l}4600 \\
4670 \\
4770 \\
\end{array}$ & snowfall & \multirow{2}{*}{$27^{\circ} 25^{\prime} \mathrm{N}$} & \multirow{2}{*}{$100^{\circ} 10^{\prime} \mathrm{E}$} & $\begin{array}{l}11 \\
44 \\
27\end{array}$ & \multirow{2}{*}{26.2} \\
\hline & $\begin{array}{l}\text { Aug } 1982 \\
\text { Aug } 1982\end{array}$ & $\begin{array}{l}3460 \\
3460 \\
\end{array}$ & rainfall & & & $\begin{array}{l}21 \\
28\end{array}$ & \\
\hline $\begin{array}{l}\text { Lenglongling } \\
\text { glacier }\end{array}$ & Aug 1982 & 4260 & snowfall & & & 196.0 & 196.0 \\
\hline
\end{tabular}

TABLE VI. THE PERCENTAGE OF GLACIAL MELTWATER IN GLACIER No 1 AT THE SOURCE OF URUMQI RIVER

\begin{tabular}{lcccccc}
\hline Months & $\begin{array}{c}\text { Tritium } \\
\text { content } \\
(\mathrm{TU})\end{array}$ & $\begin{array}{c}\text { Glacial } \\
\text { meltwater } \\
(\mathrm{TU})\end{array}$ & $\begin{array}{c}\text { Run-off } \\
\text { volume } \\
(\mathrm{TU})\end{array}$ & $\begin{array}{l}\text { Run-off } \\
\text { volume } \\
\left(\mathrm{cm}^{3} \mathrm{~s}^{-1}\right)\end{array}$ & $\begin{array}{c}\text { Glacial } \\
\text { meltwater } \\
(\%)\end{array}$ & $\begin{array}{c}\text { Snowfall } \\
(\%)\end{array}$ \\
\hline May 1983 & 79 & 50 & 66 & 0.009 & 18.9 & 81.8 \\
\hline June 1983 & 128 & 83 & 114 & 0.067 & 31.3 & 68.7 \\
\hline July 1983 & 83 & 50 & 69 & 0.400 & 42.0 & 58.0 \\
\hline Aug 1983 & 120 & 50 & 106 & 0.034 & 20.0 & 80.0 \\
\hline Mean & 113 & 54 & 108 & 0.084 & 8.48 & 91.52 \\
\hline
\end{tabular}

